\title{
Pediatric gliomatosis cerebri presenting with intratumoral hemorrhage leading to poor outcome
}

\author{
Hiromasa Adachi, Masashi Kitagawa, Toshinari Kawasaki, Takafumi Wataya \\ Department of Neurosurgery, Shizuoka Children's Hospital, Shizuoka 420-8660, Japan.
}

Correspondence to: Dr. Takafumi Wataya, Department of Neurosurgery, Shizuoka Children's Hospital, 860 Urushiyama, Aoi-ku, Shizuoka 4208660, Japan. E-mail: watayatakafumi@gmail.com

\begin{abstract}
How to cite this article: Adachi $\mathrm{H}$, Kitagawa M, Kawasaki T, Wataya T. Pediatric gliomatosis cerebri presenting with intratumoral hemorrhage leading to poor outcome. J Cancer Metastasis Treat 2016;2:375-8.
\end{abstract}

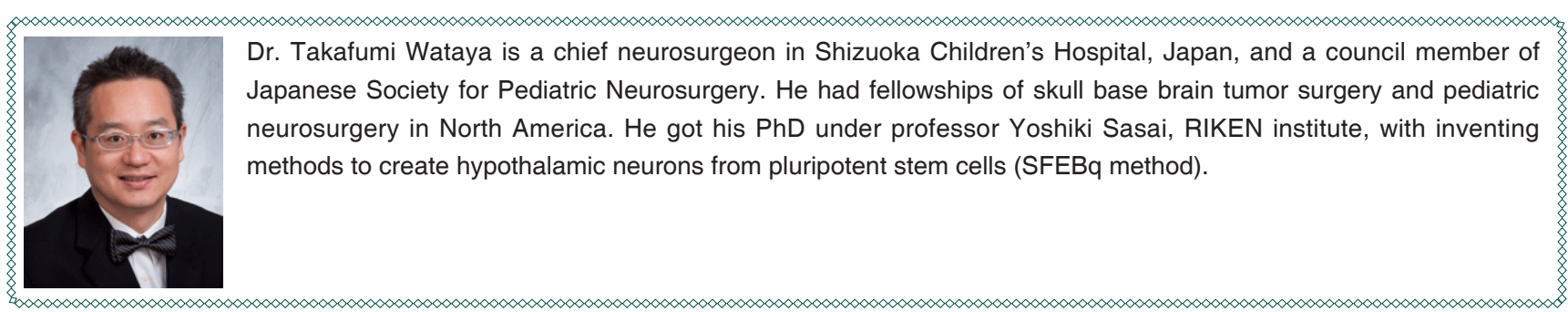

Article history:

Received: 16-06-2016

Accepted: 23-07-2016

Published: 18-09-2016

\section{Key words:}

Gliomatosis cerebri,

intratumoral hemorrhage,

pediatric

\begin{abstract}
Gliomatosis cerebri (GC) is an uncommon disease, defined as diffuse infiltration of neoplastic glial cells involving at least three cerebral lobes. GCs in young population are rare. We described a case of 14-year-old woman with GC who did not receive any recommended treatment, because the patient's family refused. The patient had a rapid deterioration in 5 months after first symptoms due to intratumoral bleeding. This is the first case report of intratumoral bleeding after diagnosis of $\mathrm{GC}$ is made, resulting in poor outcome. GC may acquire possibility of intratumoral hemorrhage through its development.
\end{abstract}

\section{INTRODUCTION}

Gliomatosis cerebri (GC) is an uncommon primary brain tumor that has quite malignant behavior. It is characterized by diffuse infiltration of glioma cells, and defined with tumor invasion into more than three cerebral lobes. ${ }^{[1,2]}$ It often infiltrates into bilateral hemispheres, in some cases, even into the brainstem, 
cerebellum, and spinal cord, affecting both gray and white matter. It is classified as grade IV in World Health Organization 2007 criteria, regardless of its histopathological features.

GCs in most cases are seen in the adult population, rarely suffering young age group. There are two peaks of patients' age distribution in the second decades and forties. ${ }^{[3]}$

We report here a pediatric patient with GC who refused any treatment, subsequently followed by rapid deterioration with intratumoral bleeding.

\section{CASE REPORT}

A 14-year-old woman presented with generalized tonic-clonic seizures following to history of morning headache, and mild cognitive deteriorations. She had noticed slowly progressing weakness on her right face and upper extremity, and numbness on her right side.

Neurological examination on admission revealed right facial droop and pronator drift on the right side. The patient originally was right-handed active softball player, but grasping power was weak with $21 \mathrm{~kg}$ on the right and $29 \mathrm{~kg}$ on the left at the time. Decreased proprioception and touch sensation was observed both in upper and lower extremities on the right. She was previously healthy and achieved normal developmental milestones and scholastic achievement up to an onset, but had experienced a decline in cognition. Wechsler Intelligence Scale for Children (WISC)-IV score shows intelligence quotient (IQ) 50.

Fluid-attenuated inversion recovery (FLAIR) and T2wighted sequences of magnetic resonance imaging (MRI) showed hyperintense signal lesion in the white matter of the left frontal, parietal, and temporal lobes [Figure $1 \mathrm{~A}$ and $1 \mathrm{~B}$ ]. Follow up MRI in 2 months later showed development of the lesion into the frontal lobe, as well as the brainstem and corpus callosum [Figure $1 \mathrm{C}$ and 1D]. MR spectroscopy at the time shows high peaks in both chorine (Cho)/N-acetilaspartate (NAA) (2.9) and Cho/Creatine (Cr) (2.46), suggesting high grade glioma [Figure 1E].

Open biopsy was performed targeting occipitotemporal mass near posterior horn of the left lateral ventricle, which shows solid swelling without contrast enhancement on MRI. Hematoxilin and eosine staining of specimen shows marked cellularity, with marked hyperchromatism and pleomorphism [Figure $2 \mathrm{~A}]$. Neither necrosis nor vascular proliferation was detected. Tumor cell infiltration in the peripheral zone of a tumor was found [Figure 2B]. Immunohistochemistry revealed positive staining for glial fibrillary acidic protein, and nuclear staining of $\mathrm{p} 53$. MIB-1 proliferation index was about $50 \%$ [Figure $2 \mathrm{C}$ and $2 \mathrm{D}$ ]. With these results, histopathological diagnosis was made as anaplastic astrocytoma (grade III). The final clinical diagnosis was determined as gliomatosis cerebri due to invasion into 3 cerebral lobes and brainstem.

Considering potential poor prognosis of the disease, the patient's parents refused either radiation or chemotherapy, and only oral corticosteroid and rehabilitation was given to the patient. Five months after
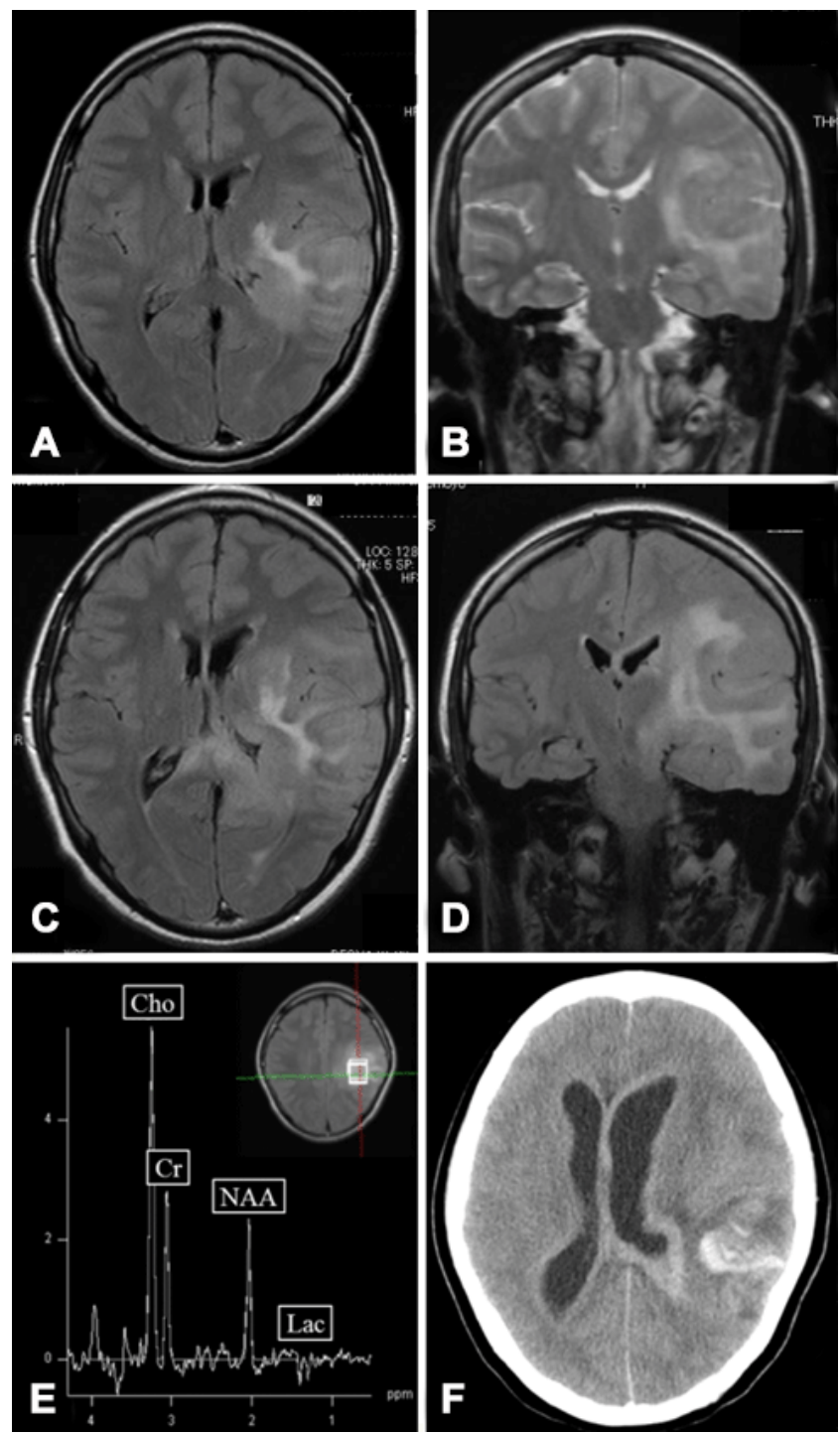

Figure 1: FLAIR and T2 weighted (T2WI) MR images demonstrating hyperintense area into 3 cerebral lobes, corpus callosum, and brainstem (A: axial FLAIR; B: coronal T2WI); follow-up FLAIR MRI took 2 months after initial symptoms (C: axial; D: coronal); MR spectroscopy suggesting high-grade glioma (E); axial CT scan demonstrating intratumoral hemorrhage 5 months after initial symptoms (F). FLAIR: fluid-attenuated inversion recovery; MRI: magnetic resonance imaging; CT: computed tomography 


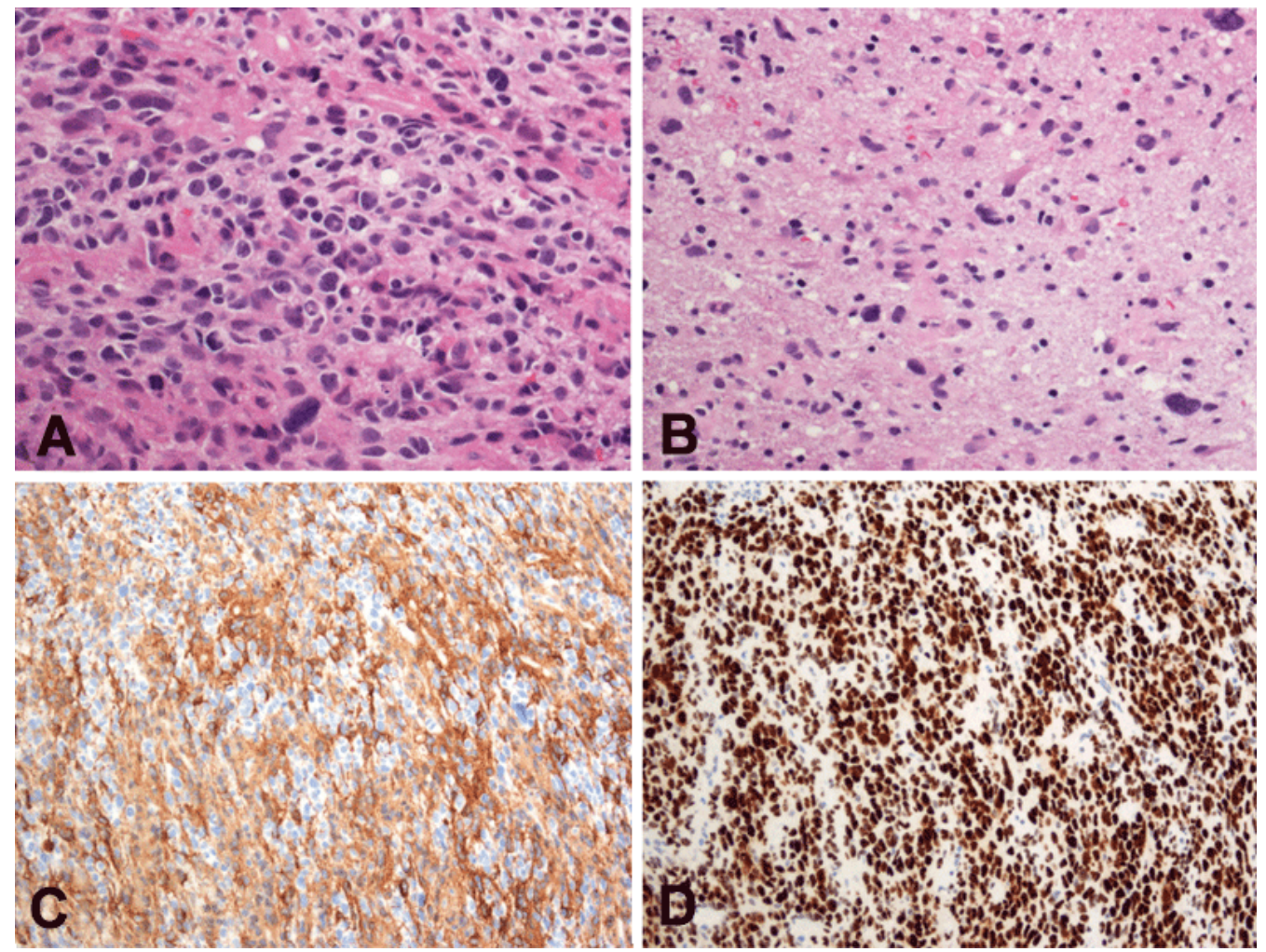

Figure 2: Hematoxilin and eosine staining of biopsy specimen. Diffuse cellular proliferation with hyperchromatism and pleomorphism (A, $\mathrm{x} 400$ ); neither necrosis nor vascular proliferation was detected. Infiltration into brain parenchyma was found (B, x400); positive staining for glial fibrillary acidic protein (C, x200), and p53 (D, x200)

the initial diagnosis, the patient had sudden respiratory arrest with uncal herniation due to intratumoral bleeding, and deceased [Figure 1F].

\section{DISCUSSION}

A case of pediatric GC which had poor clinical course with fatal intratumoral bleeding is presented. Although GCs in pediatric ages are rare, and there are no reports with large patients groups, a report with $13 \mathrm{GC}$ patients under 18 years old showed that 2 years survival rate is $67 \%$, and median overall survival is 27 months. ${ }^{[4]}$ The report also shows 2 years survival rate of patients under 10 years old is only $19 \%$. As such, pediatric GC has extremely poor prognosis.

As for treatment of GC in general, whole brain radiation therapy (WBRT) with 45-50 Gy is considered as standard therapy. ${ }^{[2]}$ Retrospective study of WBRT with $54.9 \mathrm{~Gy}$ in average shows improvement of both overall survival (OS: 27.5 vs. 6.5 months) and progression free survival (PFS: 16.5 vs. 4.5 months). ${ }^{[2]}$

Chemotherapy for GC had not been considered as effective treatments even combined with radiation therapy $(\mathrm{RT}),{ }^{\left[{ }^{5]}\right]}$ but recent growing publications support its efficacy to certain extent. ${ }^{[6]}$ Chemotherapy with temozolomide is widely used recently, because of its safety. It has, however, insufficient effect to GC, and combination with RT is considered essential. Therefore, RT is recommended even for children who potentially have higher susceptibility for radiation. ${ }^{[6]}$

In the present case, the patient deceased in 5 months after initial diagnosis, which is just as short as reported in the study in the group without WBRT. ${ }^{[2]}$ This case supports the necessity of RT in order to accomplish better OS or PFS.

Another reason why this patients had rapid deterioration was intratumoral bleeding. GC is classified into 2 types; type 1 (classical GC) shows infiltration of gliomatous cells with no mass lesions, and type 2 is categorized ones which develop tumor mass after type 1 infiltration. ${ }^{[7]}$ In the MRI of GC type 1, it has no or very small tumor enhancement with gadolinium and low relative cerebral blood volume (rCBV) value in perfusion study. ${ }^{[8,9]}$ Both indicate that the tumor has low vascularity; therefore it is expected to have small chance of intratumoral hemorrhage. To our knowledge, there is no report of intratumoral hemorrhage of type $1 \mathrm{GC}$. On the other hand, contrast enhancement can be seen in some cases of type $2 \mathrm{GC}$, with increased rCBV. ${ }^{[9]}$ From these aspects, type 2 GC may have higher possibility of bleeding than type 1 . 
This is the first case report of intratumoral hemorrhage of $\mathrm{GC}$ after its diagnosis is made. Although not frequent, the possibility of intratumoral hemorrhage of GC must be kept in mind, especially when no treatment was given, or once it become uncontrollable even with RT or chemotherapy.

A pediatric case of $\mathrm{GC}$ with intratumoral bleeding is reported. GC may acquire possibility of intratumoral hemorrhage through its development, and may lead to catastrophic outcome.

\section{Financial support and sponsorship}

Nil.

\section{Conflicts of interest}

There are no conflicts of interest.

\section{Patient consent}

Obtained.

\section{Ethics approval}

The patient was treated within the standards of authors' institute and the report was approved.

\section{REFERENCES}

1. Rudà R, Bertero L, Sanson M. Gliomatosis cerebri: a review. Curr Treat Options Neurol 2014;16:273.

2. Chen S, Tanaka S, Giannini C, Morris J, Yan ES, Buckner J, Lachance DH, Parney IF. Gliomatosis cerebri: clinical characteristics, management, and outcomes. J Neurooncol 2013;112:267-75.

3. Artigas J, Cervos-Navarro J, Iglesias JR, Ebhardt G. Gliomatosis cerebri: clinical and histological findings. Clin Neuropathol 1985;4:135-48.

4. Armstrong GT, Phillips PC, Rorke-Adams LB, Judkins AR, Localio AR, Fisher MJ. Gliomatosis cerebri: 20 Years of experience at the Children's Hospital of Philadelphia. Cancer 2006;107:1597-606.

5. Perkins GH, Schomer DF, Fuller GN, Allen PK, Maor MH. Gliomatosis cerebri: improved outcome with radiotherapy. Int J Radiat Oncol Biol Phys 2003;56:1137-46.

6. Sanson M, Cartalat-Carel S, Taillibert S, Napolitano M, Djafari L, Cougnard J, Gervais H, Laigle F, Carpentier A, Mokhtari K, Taillandier L, Chinot O, Duffau H, Honnorat J, Hoang-Xuan K, Delattre JY. Initial chemotherapy in gliomatosis cerebri. Neurology 2004;63:270-5.

7. Park S, Suh YL, Nam DH, Kim ST. Gliomatosis cerebri clinicopathologic study of 33 cases and comparison of mass forming and diffuse types. Clin Neuropathol 2009;28:73-82.

8. Yang S, Wetzel S, Law M, Zagzag D, Cha S. Dynamic contrastenhanced $\mathrm{T} 2 *$-weighted MR imaging of gliomatosis cerebri. AJNR Am J Neuroradiol 2002;23:350-5.

9. Desclée P, Rommel D, Hernalsteen D, Godfraind C, de Coene B, Cosnard G. Gliomatosis cerebri, imaging findings of 12 cases. $J$ Neuroradiol 2010;37:148-58. 